Network Working Group

Request for Comments: 870

Obsoletes RFCs: 820 ,

$790,776,770,762,758,755$,

$750,739,604,503,433,349$

Obsoletes IENs: 127, 117, 93
J. Reynolds

J. Postel

ISI

October 1983

\title{
ASSIGNED NUMBERS
}

This Network Working Group Request for Comments documents the currently assigned values from several series of numbers used in network protocol implementations. This RFC will be updated periodically, and in any case current information can be obtained from Joyce Reynolds. The assignment of numbers is also handled by Joyce. If you are developing a protocol or application that will require the use of a link, socket, port, protocol, or network number please contact Joyce to receive a number assignment.

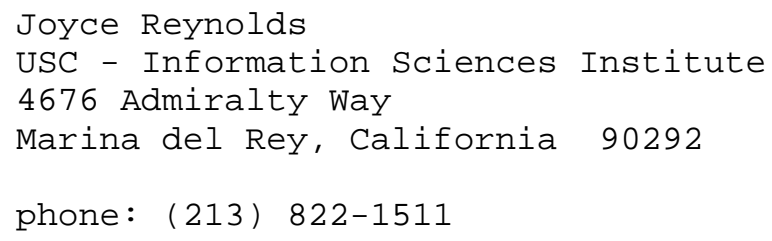

Most of the protocols mentioned here are documented in the RFC series of notes. The more prominent and more generally used are documented in the "Internet Protocol Transition Workbook" [16] or in the old "ARPANET Protocol Handbook" [17] prepared by the NIC. Some of the items listed are undocumented. Further information on protocols can be found in the memo "Official Protocols" [52].

In all cases the name and mailbox of the responsible individual is indicated. In the lists that follow, a bracketed entry, e.g., [16,iii], at the right hand margin of the page indicates a reference for the listed protocol, where the number cites the document and the "iii" cites the person. 
The network numbers listed here are used as internet addresses by the Internet Protocol (IP) [16,47]. The IP uses a 32-bit address field and divides that address into a network part and a "rest" or local address part. The division takes 3 forms or classes.

The first type of address, or class A, has a 7-bit network number and a 24-bit local address. The highest-order bit is set to 0 . This allows 128 class A networks.

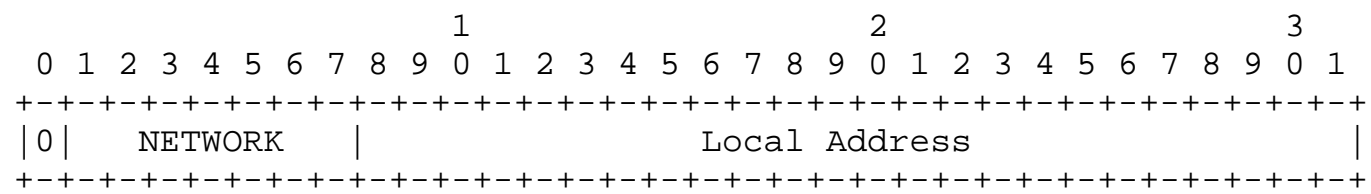

Class A Address

The second type of address, class B, has a 14-bit network number and a 16-bit local address. The two highest-order bits are set to 1-0. This allows 16,384 class B networks.

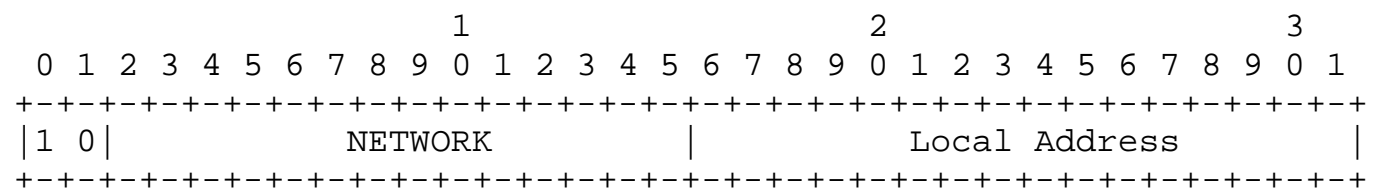

The third type of address, class C, has a 21-bit network number and a 8-bit local address. The three highest-order bits are set to 1-1-0. This allows 2,097,152 class C networks.

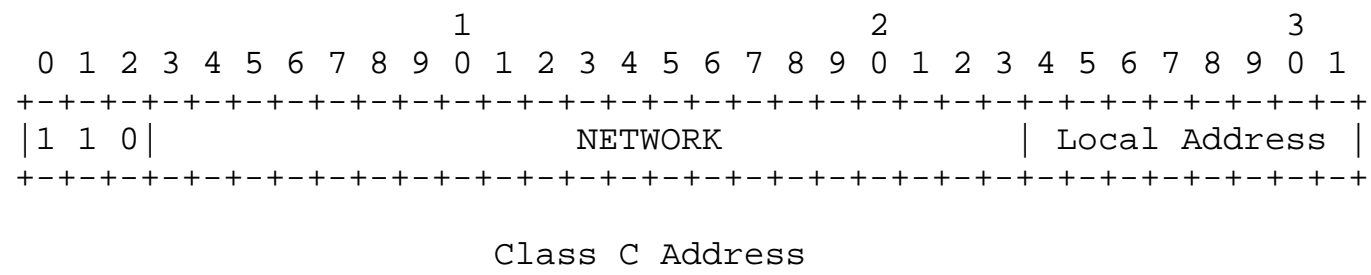

Note: No addresses are allowed with the three highest-order bits set to 1-1-1. These addresses (sometimes called "class D") are reserved. 
One commonly used notation for internet host addresses divides the 32-bit address into four 8-bit fields and specifies the value of each field as a decimal number with the fields separated by periods. This is called the "dotted decimal" notation. For example, the internet address of ISIF in dotted decimal is 010.002.000.052, or 10.2.0.52.

The dotted decimal notation will be used in the listing of assigned network numbers. The class A networks will have nnn.rrr.rrr.rrr, the class B networks will have nnn.nnn.rrr.rrr, and the class C networks will have nnn.nnn.nnn.rrr, where nnn represents part or all of a network number and rrr represents part or all of a local address.

There are three catagories of users of Internet Addresses: Research, Defense, and Commercial. To reflect the allocation of network identifiers among the categories, a one-character code is placed to the left of the network number (in the column marked by an asterisk): $\mathrm{R}$ for Research and Development, $\mathrm{D}$ for DoD, and C for Commercial.

Numbers assigned for commercial use of IP family protocols, but not for interworking with the ARPA or DoD Internets are marked with an astrisk preceeding the number.

For various reasons, the assigned numbers of networks are sometimes changed. To ease the transition the old number will be listed as well. These "old number" entries will be marked with a "T" following the number and preceeding the name, and the network name will be the suffixed "-TEMP". 
Assigned Network Numbers

Class A Networks

\begin{tabular}{|c|c|c|c|}
\hline \multirow{3}{*}{ - } & Internet Address & Name & References \\
\hline & ---------------- & ---- & ------- \\
\hline & 000 .rrr.rrr.rrr & & Reserved \\
\hline & 003 .rre.rrr.rrr & RCC-NET-TEMP & BBN RCC Network \\
\hline & $04 . r r r . r r r . r r r$ & SATNET & Atlantic Satellite Network [DM] \\
\hline & $05 . \operatorname{rrr}$.rrr.rrr & DEMO-PR-1-TEMP & Demo-1 Packet Radio Network [LC \\
\hline & $06 . r r r . r r r . r r r$ & T YPG-NET-TEMP & Yuma Proving Grounds $\quad[2, \mathrm{BX}$ \\
\hline & $07 . r r r . r r r . r r r$ & $\mathrm{~T}$ EDN-TEMP & DCEC EDN \\
\hline & $08 . r r r . r r r . r r r$ & T BBN-NET-TEMP & BBN Network \\
\hline & 09.rrr.rrr.rrr & T BRAGG-PR-TEMP & Ft. Bragg Packet Radio Net [JE] \\
\hline & 10.rrr.rrr.rrr & ARPANET & ARPANET $\quad[2,17, \mathrm{REK}$ \\
\hline C & 012 .rrr.rrr.rrr & $\mathrm{ATT}$ & ATT, Bell Labs \\
\hline 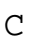 & 14.rrr.rrr.rer & $\mathrm{PDN}$ & Public Data Network \\
\hline & $018 . r r r . r r r . r r r$ & T MIT-TEMP & MIT Network \\
\hline & $023 . r r r . r r r . r r r$ & MITRE & MITRE Cablenet \\
\hline & 024 .rrr.rrr.rrr & MINET & MINET \\
\hline & $025 . \operatorname{rrr}$.rrr.rrr & RSRE-EXP & RSRE Experimental \\
\hline & 026.rrr.rrr.rrr & MILNET & MILNET \\
\hline & 027 .rrr.rrr.rrr & T NOSC-LCCN-TEMP & NOSC / LCCN \\
\hline 2 & $028 . r r r . r r r . r r r$ & WIDEBAND & Wide Band Satellite Net \\
\hline & 032 .rrr.rrr.rrr & $\mathrm{UCL}-\mathrm{TAC}$ & $\mathrm{UCL}$ TAC \\
\hline & 035.rrr.rrr.rrr & T RSRE-NULL-TEMP & RSRE Null Network \\
\hline R & 36.rrr.rrr.rrr & T SU-NET-TEMP & Stanford University Network [ JCl \\
\hline & 039.rrr.rrr.rrr & SRINET-TEMP & SRI Local Network [GEO \\
\hline R & $041 . r r r . r r r . r r r$ & BBN-TEST-A & BBN-GATE-TEST-A \\
\hline & $044 . r r r . r r r . r r r$ & AMPRNET & Amateur Radio Experiment Net [H \\
\hline & $045 . \operatorname{rrr} . \operatorname{rrr} . \operatorname{rrr}$ & T C3-PR-TEMP & Testbed Development PRNET [BG \\
\hline 2 & $046 . r r r . r r r . r r r$ & T Berkeley-TEMP & Berkeley Ethernet \\
\hline 2 & 047 .rrr.rrr.rrr & T SAC-PR-TEMP & SAC Packet Radio Network \\
\hline 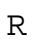 & $048 . \operatorname{rrr} . \operatorname{rrr} . \operatorname{rrr}$ & NDRE-TIU & NDRE-TIU \\
\hline & $050 . \operatorname{rrr} . \operatorname{rrr}$.rrr & NTA-RING & NDRE-RING \\
\hline & 001 .rrr.rrr.rrr -0 & 002 .rrr.rrr.rrr & Unassigned \\
\hline & $011 . \operatorname{rrr}$.rre.rrr & & signed \\
\hline & $013 . r r r . r r r . r r r$ & & signed \\
\hline & 015 .rrr.rrr.rrr -0 & 017 .rrr.rrr.rrr & signed \\
\hline & $019 . \operatorname{rrr}$.rrr.rrr-0 & 022 .rrr.rrr.rrr & signed \\
\hline & $029 . \operatorname{rrr}$.rre.rrr-0 & 031 .rrr.rrr.rrr & Unassigned \\
\hline & 033 .rrr.rrr.rrr-0 & 034 .rrr.rrr.rrr & Unassigned \\
\hline & 037 .rrr.rrr.rrr -0 & 038 .rrr.rrr.rrr & Unassigned \\
\hline & $040 . r r r . r r r . r r r$ & & Unassigned \\
\hline & 042 .rrr.rrr.rrr -0 & 043 .rrr.rrr.rrr & Unassigned \\
\hline & $049 . \operatorname{rrr}$.rrr.rrr & & Unassigned \\
\hline & 051.rrr.rrr.rrr -1 & 126.rrr.rrr.r & Unassigned \\
\hline & 127.rrr.rrr.rrr & & Reserved \\
\hline
\end{tabular}


Class B Networks

\begin{tabular}{|c|c|c|c|}
\hline & Internet Address & Name & References \\
\hline & \multirow{2}{*}{\multicolumn{2}{|c|}{$\begin{array}{l}-128.000 . \operatorname{rrr} . \operatorname{rrr} \\
128\end{array}$}} & ------- \\
\hline & & & Reserved \\
\hline & $128.001 . \operatorname{rrr} . \operatorname{rrr}$ & $\mathrm{BBN}-\mathrm{TEST}-\mathrm{B}$ & BBN-GATE-TEST-B \\
\hline & $128.002 . \operatorname{rrr} \operatorname{srr}$ & $\mathrm{CMU}-\mathrm{NET}$ & CMU-Ethernet \\
\hline & $128.003 . \operatorname{rrr} \operatorname{srr}$ & LBL-CSAM & LBL-CSAM-RESEARCH \\
\hline & $28.004 . \operatorname{rrr} . \operatorname{rrr}$ & CNET & LINKABIT DCNET \\
\hline & 5.rrr.rrr & ORDNET & FORD DCNET \\
\hline & 6.rre.rrr & RUTGERS & RUTGERS \\
\hline & 7.rre.rrr & DEVLR & DFVLR DCNET Network \\
\hline & 8.rrr.rrr & UMDNET & Univ of Maryland DCNET \\
\hline & 9.rrr.rrr & ISI-NET & USC-ISI Local Network \\
\hline & $0 . \operatorname{rrr} \operatorname{srr}$ & PURDUE-CS & Purdue Computer Science \\
\hline & 1.rre.rrr & BBN-CRONUS & BBN DOS Project $\quad[29, \pi$ \\
\hline & 2.rrr.rrr & $\mathrm{SU}-\mathrm{NET}$ & Stanford University Net \\
\hline & 3rr.rer & MATNET & Mobile Access Terminal Net [DM \\
\hline & .rre.rrr & $\mathrm{BBN}-\mathrm{SAT}-\mathrm{TEST}$ & BBN SATNET Test Net \\
\hline & .rre.rrr & S1NET & LLL-S1-NET \\
\hline & .rrr.rrr & UCLNET & University College London \\
\hline & 7.rrr.rrr & MATNET-ALT & Mobile Access Terminal Alt [DM1] \\
\hline & 8.rrr.rrr & SRINET & SRI Local Network \\
\hline & .rre.rrr & EDN & DCEC EDN \\
\hline & 0.rrr.rrr & BRLNET & BRLNET $\quad[2, \mathrm{MJM} 2$ \\
\hline & .rrr.rrr & $S F-P R-1$ & SF-1 Packet Radio Network [JEM \\
\hline & $22 . \operatorname{rrr} \operatorname{srr}$ & $S F-P R-2$ & SF-2 Packet Radio Network \\
\hline & 3.rre.rrr & $\mathrm{BBN}-\mathrm{PR}$ & BBN Packet Radio Network \\
\hline & .rrr.rrr & ROCK & Rockwell Packet Radio Net \\
\hline & .rre.rrr & BRAG & Ft. Bragg Packet Radio Net \\
\hline & .rre.rrr & $S A C-P R$ & SAC Packet Radio Network [B \\
\hline & 7.rrr.rrr & $\mathrm{DEMO}-\mathrm{PR}-1$ & Demo-1 Packet Radio Network [ LC \\
\hline ) & $\begin{array}{l}\text { 3.rrr.rrr } \\
\text { 9.rrr.rrr }\end{array}$ & $\mathrm{C} 3-\mathrm{PR}$ & $\begin{array}{l}\text { Testbed Development PR NET } \\
\text { Unassigned }\end{array}$ \\
\hline & $0 . r r r . r r r$ & MIT-NET & MIT Local Network \\
\hline 2 & 1.rrr.rrr & MIT-RES & MIT Research Network \\
\hline & 2.rrr.rrr & UCB-ETHER & UC Berkeley Ethernet \\
\hline R & 3.rrr.rrr & $\mathrm{BBN}-\mathrm{NET}$ & BBN Network \\
\hline & 4.rrr.rrr & NOSC-LCCN & NOSC / LCCN \\
\hline & 5.rrr.rrr & CISLTESTNET1 & Honeywell \\
\hline 2 & 36.rrr.rrr & YALE-NET & YALE NET \\
\hline & 37.rrr.rrr & YPG-NET & Yuma Proving Grounds \\
\hline & $128.038 . \operatorname{rrr} . \operatorname{rrr}$ & NSWC-NET & NSWC Local Host Net \\
\hline & $128.039 . \operatorname{rrr} . \operatorname{rrr}-$ & $1.254 . \operatorname{rrr}$ & Unassigned \\
\hline & $191.255 . \operatorname{rrr} . \operatorname{rrr}$ & & Reserved \\
\hline
\end{tabular}




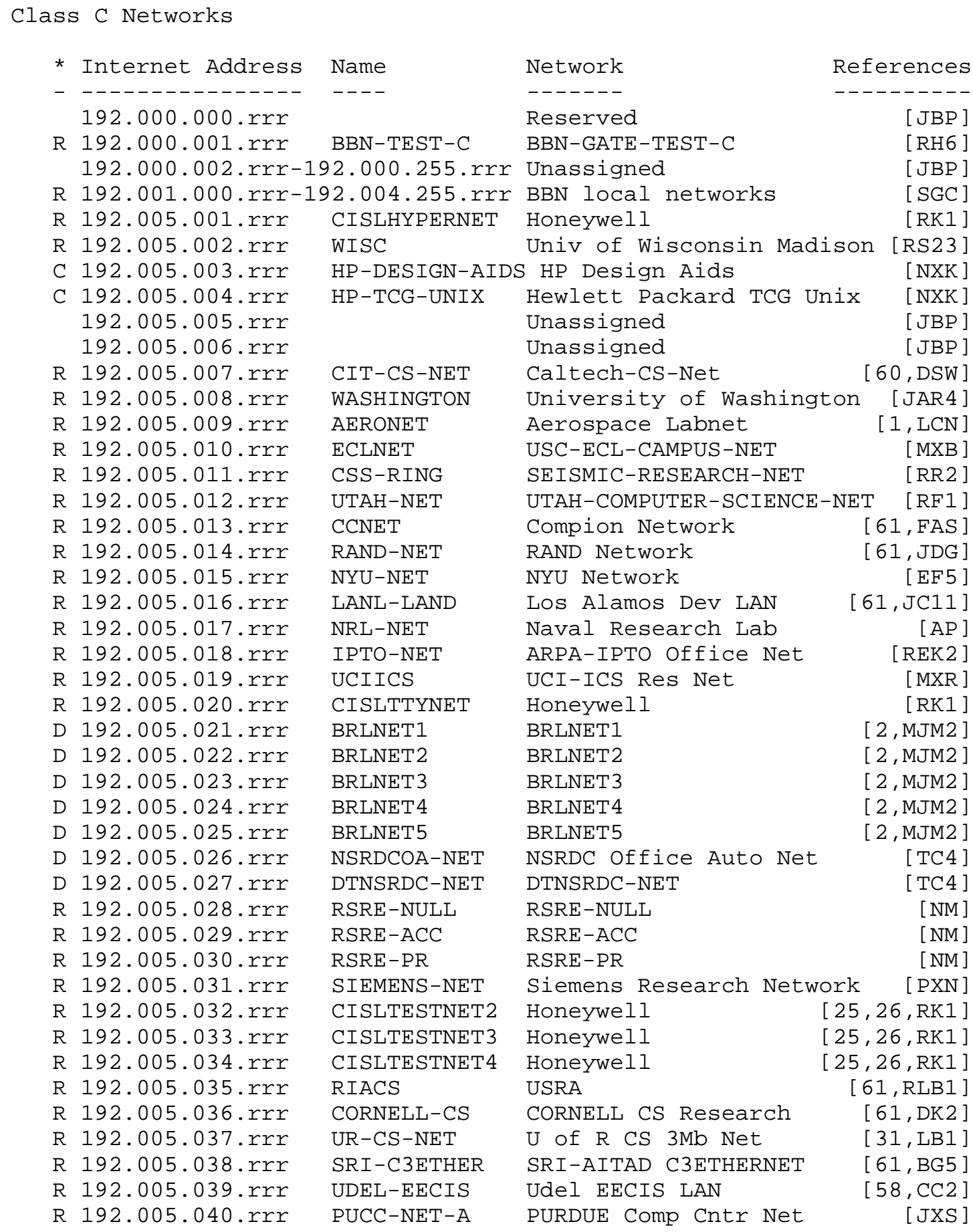




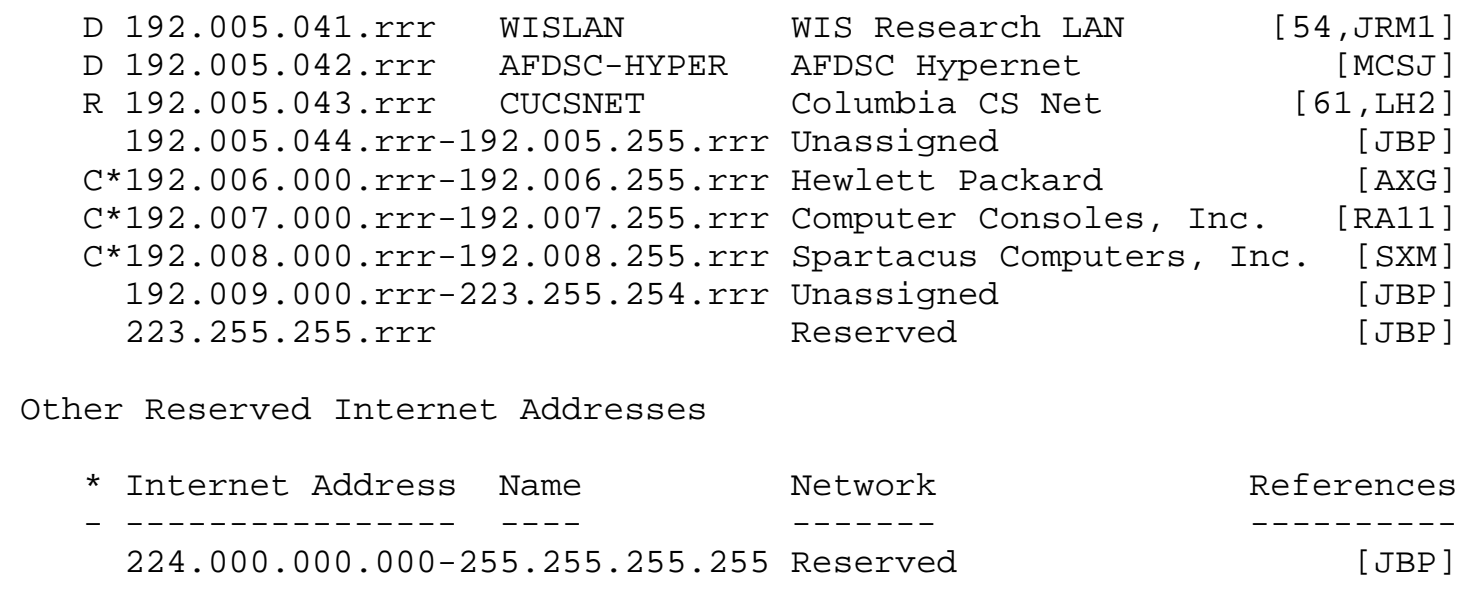


Network Numbers

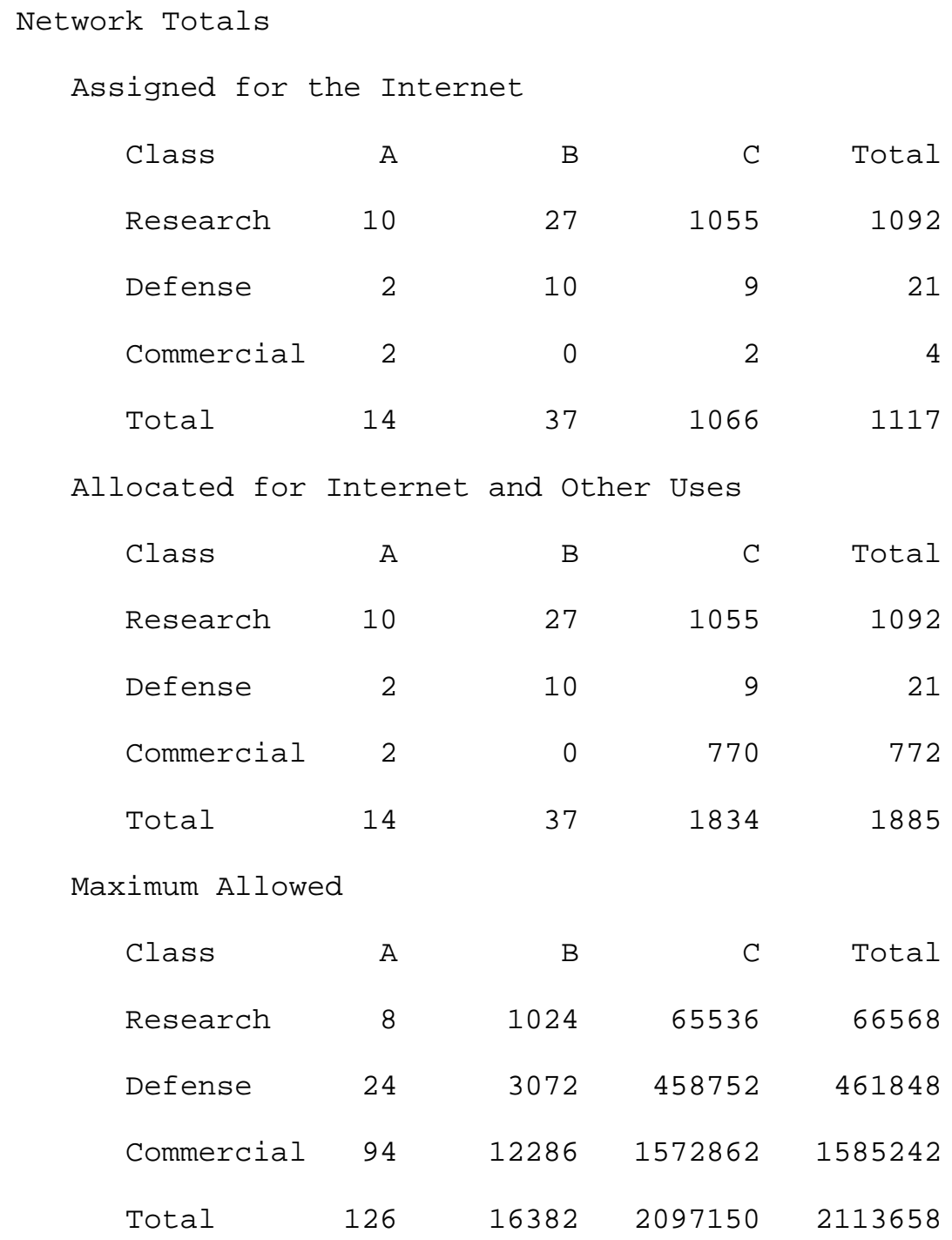


In the Internet Protocol (IP) [16,47] there is a field to identify the version of the internetwork general protocol. This field is 4 bits in size.

Assigned Internet Version Numbers

$\begin{array}{rclr}\text { Decimal } & \text { Octal } & \text { Version } & \text { References } \\ ----- & ----- & ----- & ------- \\ 0 & 0 & \text { Reserved } & \text { [ JBP ] } \\ 1-3 & 1-3 & \text { Unassigned } & \text { [ JBP ] } \\ 4 & 4 & \text { Internet Protocol } & \text { [16, 47, JBP] } \\ 5 & 5 & \text { ST Datagram Mode } & \text { [18, JWF] } \\ 6-14 & 6-16 & \text { Unassigned } & \text { [ JBP ] } \\ 15 & 17 & \text { Reserved } & \text { [ JBP ] }\end{array}$

ASSIGNED INTERNET PROTOCOL NUMBERS

In the Internet Protocol (IP) [16,47] there is a field, called Protocol, to identify the the next level protocol. This is an 8 bit field.

Assigned Internet Protocol Numbers

\begin{tabular}{|c|c|c|c|}
\hline Decimal & Octal & Protocol & References \\
\hline------- & ----- & ---------------- & ---------- \\
\hline 0 & 0 & Reserved & {$[\mathrm{JBP}]$} \\
\hline 1 & 1 & ICMP & {$[16,40, \mathrm{JBP}]$} \\
\hline 2 & 2 & Unassigned & {$[\mathrm{JBP}]$} \\
\hline 3 & 3 & Gateway-to-Gateway & {$[24, \mathrm{MB}]$} \\
\hline 4 & 4 & Unassigned & [ JBP ] \\
\hline 5 & 5 & Stream (ST) & {$[18, \mathrm{JWF}]$} \\
\hline 6 & 6 & Transmission Control (TCP) & {$[16,48, \mathrm{JBP}]$} \\
\hline 7 & 7 & $\mathrm{UCL}$ & {$[\mathrm{PK}]$} \\
\hline 8 & 10 & Exterior Gateway Protocol (EGP) & {$[53, \mathrm{RH} 6]$} \\
\hline 9 & 11 & Unassigned & {$[\mathrm{JBP}]$} \\
\hline 10 & 12 & BBN RCC Monitoring & {$[\mathrm{SGC}]$} \\
\hline 11 & 13 & NVP & {$[12, \mathrm{SC} 3]$} \\
\hline 12 & 14 & PUP & {$[6, \mathrm{EAT} 3]$} \\
\hline $13-14$ & $15-16$ & Unassigned & {$[\mathrm{JBP}]$} \\
\hline 15 & 17 & Cross Net Debugger (XNET) & {$[23, \mathrm{JFH} 2]$} \\
\hline 16 & 20 & Chaos Stream & {$[\mathrm{NC} 3]$} \\
\hline 17 & 21 & User Datagram (UDP) & {$[16,46, \mathrm{JBP}]$} \\
\hline 18 & 22 & Multiplexing & {$[13, \mathrm{JBP}]$} \\
\hline 19 & 23 & DCN Measurement Subsystems & [DLM1 ] \\
\hline 20 & 24 & Host Monitoring (HMP) & {$[28, \mathrm{RH} 6]$} \\
\hline 21 & 25 & Packet Radio Measurement & {$[\mathrm{ZSU}]$} \\
\hline
\end{tabular}


Internet Protocol Numbers

$\begin{array}{rr}22 & 26 \\ 23 & 27 \\ 24 & 30 \\ 25 & 31 \\ 26 & 32 \\ 27-60 & 33-74 \\ 61 & 75 \\ 62 & 76 \\ 63 & 77 \\ 64 & 100 \\ 65 & 101 \\ 66 & 102 \\ 67 & 103 \\ 68 & 104 \\ 69 & 105 \\ 70 & 106 \\ 71 & 107 \\ 72-75 & 110-113 \\ 76 & 114 \\ 77 & 115 \\ 78 & 116 \\ 79 & 117 \\ 80-254 & 120-376 \\ 255 & 377\end{array}$

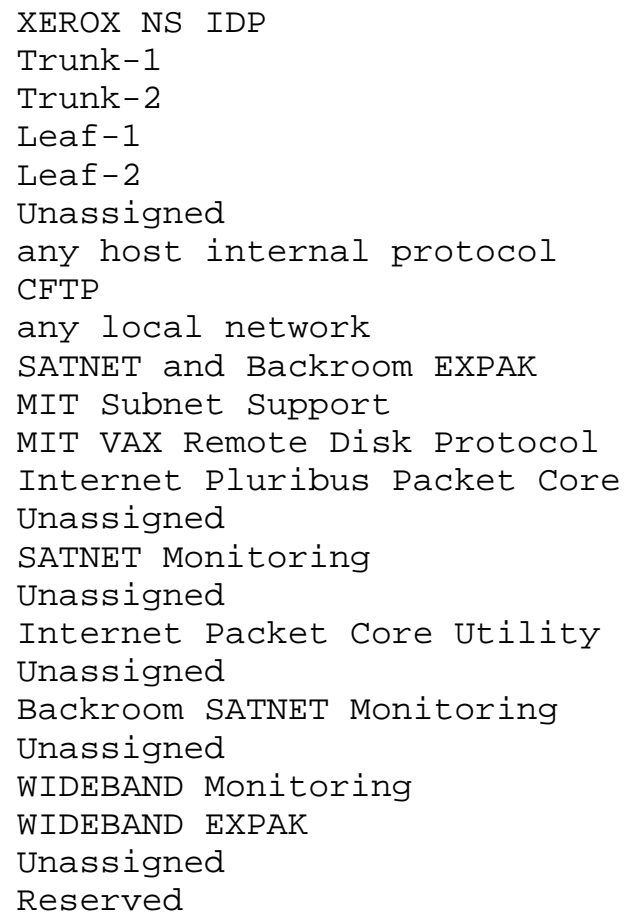


Ports are used in the $\operatorname{TCP}[16,48]$ to name the ends of logical connections which carry long term conversations. For the purpose of providing services to unknown callers, a service contact port is defined. This list specifies the port used by the server process as its contact port. The contact port is sometimes called the "well-known port".

To the extent possible, these same port assignments are used with the $\operatorname{UDP}[16,46]$.

The assigned ports use a small portion of the possible port numbers. The assigned ports have all except the low order eight bits cleared to zero. The low order eight bits are specified here.

Port Assignments:

\begin{tabular}{|c|c|c|c|}
\hline Decimal & Octal & Description & References \\
\hline------- & ----- & ----------- & ---------- \\
\hline 0 & 0 & Reserved & {$[\mathrm{JBP}]$} \\
\hline $1-4$ & $1-4$ & Unassigned & [ JBP ] \\
\hline 5 & 5 & Remote Job Entry & {$[8,17, \mathrm{JBP}]$} \\
\hline 7 & 7 & Echo & {$[38, \mathrm{JBP}]$} \\
\hline 9 & 11 & Discard & {$[37, \mathrm{JBP}]$} \\
\hline 11 & 13 & Active Users & {$[34, \mathrm{JBP}]$} \\
\hline 13 & 15 & Daytime & {$[36, \mathrm{JBP}]$} \\
\hline 15 & 17 & Who is up or NETSTAT & {$[\mathrm{JBP}]$} \\
\hline 17 & 21 & Quote of the Day & {$[43, \mathrm{JBP}]$} \\
\hline 19 & 23 & Character Generator & {$[35, \mathrm{JBP}]$} \\
\hline 20 & 24 & File Transfer (Default Data) & {$[16,39, \mathrm{JBP}]$} \\
\hline 21 & 25 & File Transfer (Control) & {$[16,39, \mathrm{JBP}]$} \\
\hline 23 & 27 & Telnet & {$[50, \mathrm{JBP}]$} \\
\hline 25 & 31 & SMTP & {$[16,45, \mathrm{JBP}]$} \\
\hline 27 & 33 & NSW User System FE & {$[14, \mathrm{RHT}]$} \\
\hline 29 & 35 & MSG ICP & {$[32, \mathrm{RHT}]$} \\
\hline 31 & 37 & MSG Authentication & {$[32, \mathrm{RHT}]$} \\
\hline 33 & 41 & Unassigned & {$[\mathrm{JBP}]$} \\
\hline 35 & 43 & Any Printer Server & [ JBP ] \\
\hline 37 & 45 & Time & {$[49, \mathrm{JBP}]$} \\
\hline 39 & 47 & Unassigned & {$[\mathrm{JBP}]$} \\
\hline 41 & 51 & Graphics & {$[17,57, \mathrm{JBP}]$} \\
\hline 42 & 52 & Host Name Server & {$[16,42, \mathrm{JBP}]$} \\
\hline 43 & 53 & NICNAME (WhoIs) & {$[16,22, \mathrm{JAKE}]$} \\
\hline 44 & 54 & MPM FLAGS Protocol & {$[\mathrm{JBP}]$} \\
\hline 45 & 55 & Message Processing Module (receive) & {$[41, \mathrm{JBP}]$} \\
\hline 46 & 56 & MPM (default send) & {$[41, \mathrm{JBP}]$} \\
\hline 47 & 57 & NI FTP & {$[59, \mathrm{SK}]$} \\
\hline
\end{tabular}


Port Numbers

\begin{tabular}{|c|c|c|c|}
\hline 49 & 61 & Login Host Protocol & {$[\mathrm{PXD}]$} \\
\hline 51 & 63 & IMP Logical Address Maintenance & {$[30, \mathrm{AGM}]$} \\
\hline 53 & 65 & Domain Name Server & [PM1 ] \\
\hline 55 & 67 & ISI Graphics Language & {$[5, \mathrm{RB} 6]$} \\
\hline 57 & 71 & Any Private Terminal Access & {$[\mathrm{JBP}]$} \\
\hline 59 & 73 & Any Private File Service & [ JBP ] \\
\hline 61 & 75 & NIMAIL & {$[3, \mathrm{SK}]$} \\
\hline 63 & 77 & Unassigned & [ JBP ] \\
\hline 65 & 101 & Unassigned & {$[\mathrm{JBP}]$} \\
\hline 67 & 103 & Datacomputer at CCA & {$[10, \mathrm{JZS}]$} \\
\hline 69 & 105 & Trivial File Transfer & {$[16,55, \mathrm{KRS}]$} \\
\hline 71 & 107 & NETRJS & {$[7,17, \mathrm{RTB}]$} \\
\hline 72 & 110 & NETRJS & {$[7,17, \mathrm{RTB}]$} \\
\hline 73 & 111 & NETRJS & {$[7,17, \mathrm{RTB}]$} \\
\hline 74 & 112 & NETRJS & {$[7,17, \mathrm{RTB}]$} \\
\hline 75 & 113 & Any Private Dial Out Service & {$[\mathrm{JBP}]$} \\
\hline 77 & 115 & Any Private RJE Service & {$[\mathrm{JBP}]$} \\
\hline 79 & 117 & Finger (Name) & {$[17,20, \mathrm{KLH}]$} \\
\hline 81 & 121 & HOSTS2 Name Server & {$[$ EAK1 ] } \\
\hline 83 & 123 & MIT ML Device & [DPR ] \\
\hline 85 & 125 & MIT ML Device & {$[\mathrm{DPR}]$} \\
\hline 87 & 127 & any terminal link & {$[\mathrm{JBP}]$} \\
\hline 89 & 131 & SU/MIT Telnet Gateway & {$[\mathrm{MRC}]$} \\
\hline 91 & 133 & MIT Dover Spooler & {$[\mathrm{EBM}]$} \\
\hline 93 & 135 & Device Control Protocol & {$[\mathrm{DCT}]$} \\
\hline 95 & 137 & SUPDUP & {$[15, \mathrm{MRC}]$} \\
\hline 97 & 141 & Datacomputer status & {$[10, \mathrm{JZS}]$} \\
\hline 99 & 143 & Metagram Relay & {$[\mathrm{GEOF}]$} \\
\hline 101 & 145 & NIC Host Name Server & {$[16,21, \mathrm{JAKE}]$} \\
\hline 103 & 147 & Unassigned & {$[\mathrm{JBP}]$} \\
\hline 105 & 151 & CSNET Mailbox Name Server (Program) & {$[56, \mathrm{MHS} 1]$} \\
\hline 107 & 153 & Remote Telnet Service & {$[44, \mathrm{JBP}]$} \\
\hline $109-129$ & $155-201$ & Unassigned & {$[\mathrm{JBP}]$} \\
\hline 131 & 203 & Datacomputer & {$[10, \mathrm{JZS}]$} \\
\hline $132-223$ & $204-337$ & Reserved & {$[\mathrm{JBP}]$} \\
\hline $224-241$ & $340-361$ & Unassigned & [ JBP ] \\
\hline 243 & 363 & Survey Measurement & {$[4, \mathrm{AV}]$} \\
\hline 245 & 365 & LINK & {$[9, \mathrm{RDB} 2]$} \\
\hline $247-255$ & $367-377$ & Unassigned & {$[\mathrm{JBP}]$} \\
\hline
\end{tabular}




\section{ASSIGNED AUTONOMOUS SYSTEM NUMBERS}

The Exterior Gateway Protocol (EGP) [53] specifies that groups of gateways may form autonomous systems. The EGP provides a 16-bit field for identifying such systems. The values of this field are registered here.

Autonomous System Numbers:

$\begin{aligned} \text { Decimal } & \text { Description } \\ ----- & --------- \\ 0 & \text { Reserved } \\ 1 & \text { The BBN Gateways } \\ 2 & \text { The DCN Gateways } \\ 3 & \text { The MIT Gateways } \\ 4-65534 & \text { Unassigned } \\ 65535 & \text { Reserved }\end{aligned}$

References
$[\mathrm{JBP}]$
$[\mathrm{MB}]$
$[\mathrm{DLM1}]$
$[\mathrm{LM}$ ]
$[\mathrm{JBP}]$
$[\mathrm{JBP}]$


The word "link" here refers to a field in the original ARPANET Host/IMP interface leader. The link was originally defined as an 8-bit field. Later specifications defined this field as the "Message-ID" with a length of 12 bits. The name link now refers to the high order 8 bits of this 12-bit message-id field. The low order 4 bits of the message-id field are to be zero unless explicitly specified otherwise for the particular protocol used on that link. The Host/IMP interface is defined in BBN Report 1822 [2].

Link Assignments:

\begin{tabular}{|c|c|c|c|}
\hline Decimal & Octal & Description & References \\
\hline-------- & ----- & ----------- & ---------- \\
\hline 0 & 0 & Reserved & {$[\mathrm{JBP}]$} \\
\hline $1-149$ & $1-225$ & Unassigned & {$[\mathrm{JBP}]$} \\
\hline 150 & 226 & Xerox NS IP & {$[62, \mathrm{LLG}]$} \\
\hline 151 & 227 & Unassigned & {$[\mathrm{JBP}]$} \\
\hline 152 & 230 & PARC Universal Protocol & {$[6, \mathrm{EAT} 3]$} \\
\hline 153 & 231 & TIP Status Reporting & {$[\mathrm{JGH}]$} \\
\hline 154 & 232 & TIP Accounting & {$[\mathrm{JGH}]$} \\
\hline 155 & 233 & Internet Protocol (regular) & {$[16,47, \mathrm{JBP}]$} \\
\hline $156-158$ & $234-236$ & Internet Protocol (experimental) & {$[16,47, \mathrm{JBP}]$} \\
\hline $159-195$ & $237-303$ & Unassigned & {$[\mathrm{JBP}]$} \\
\hline $196-255$ & $304-377$ & Experimental Protocols & {$[\mathrm{JBP}]$} \\
\hline $248-255$ & $370-377$ & Network Maintenance & {$[\mathrm{JGH}]$} \\
\hline
\end{tabular}

\section{ETHERNET NUMBERS OF INTEREST}

Many of the networks of all classes are Ethernets (10Mb) or Experimental Ethernets (3Mb). These systems use a message "type" field in much the same way the ARPANET uses the "link" field.

Assignments:

\begin{tabular}{|c|c|}
\hline $\begin{array}{l}\text { Etherne } \\
------\end{array}$ & \\
\hline decimal & $\mathrm{He}$ \\
\hline 512 & 02,0 \\
\hline 1536 & 06,0 \\
\hline 2048 & 08,00 \\
\hline 2054 & 08,0 \\
\hline
\end{tabular}

$\begin{array}{rcl}\text { Exp. Ethernet } & \text { Description } \\ ----------- & --------- \\ \text { decimal } & \text { octal } & \\ 512 & 1000 & \text { XEROX PUP } \\ 1536 & 3000 & \text { XEROX NS IDP } \\ 513 & 1001 & \text { DOD IP } \\ - & - & \text { Address Res }\end{array}$

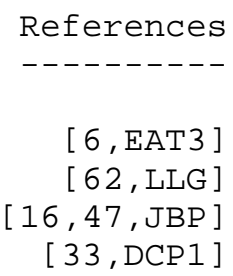




\section{ASSIGNED PUBLIC DATA NETWORK NUMBERS}

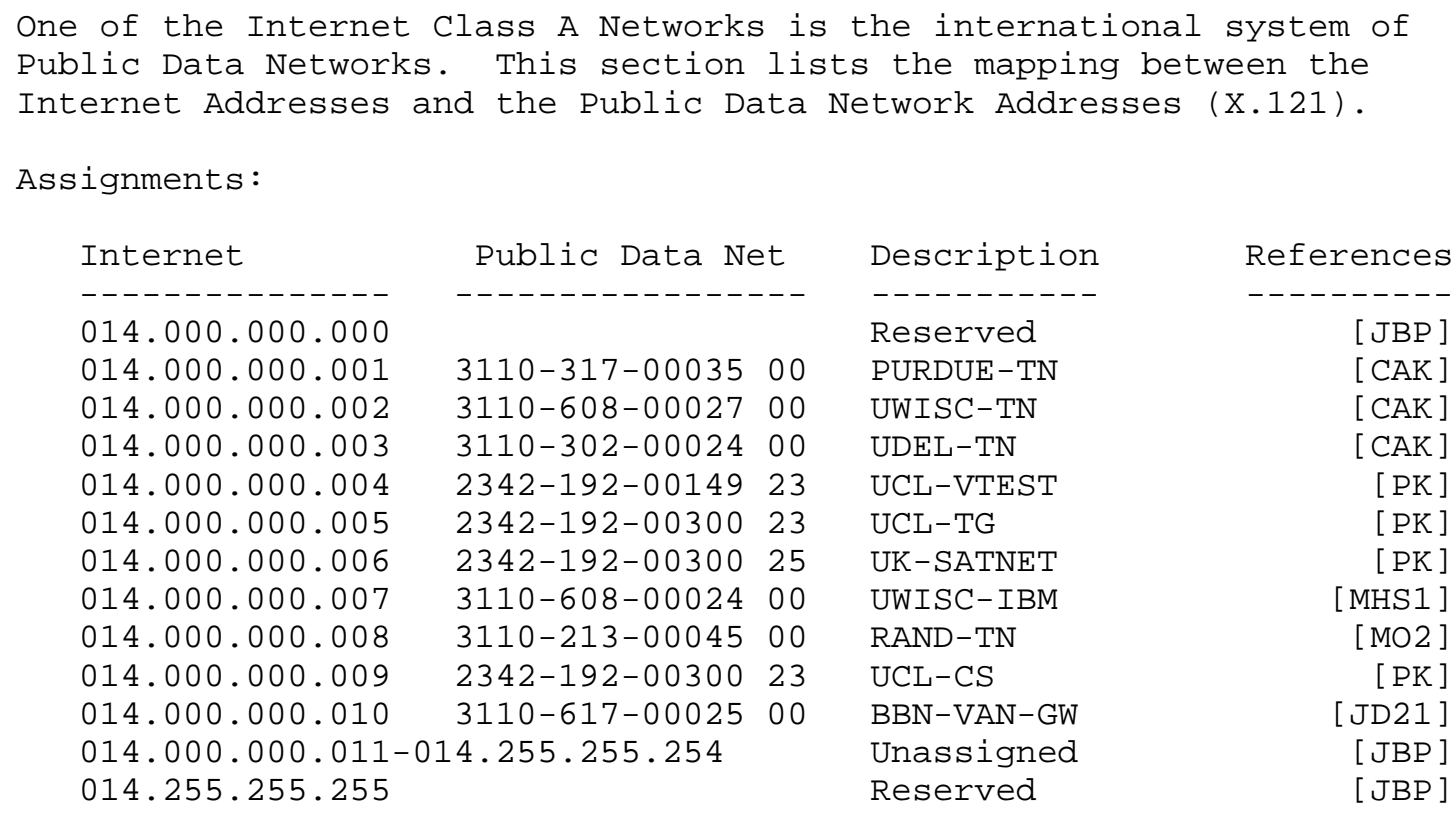

The standard for transmission of IP datagrams over the Public Data Network is specified in [27]. 
[1] Aerospace, Internal Report, ATM-83(3920-01)-3, 1982.

[2] BBN, "Specifications for the Interconnection of a Host and an IMP", Report 1822, Bolt Beranek and Newman, Cambridge, Massachusetts, revised, December 1981.

[3] Bennett, C., "A Simple NIFTP-Based Mail System", IEN 169, University College, London, January 1981.

[4] Bhushan, A., "A Report on the Survey Project", RFC 530, NIC 17375, 22 June 1973.

[5] Bisbey, R., D. Hollingworth, and B. Britt, "Graphics Language (version 2.1)", ISI/TM-80-18, USC/Information Sciences Institute, July 1980 .

[6] Boggs, D., J. Shoch, E. Taft, and R. Metcalfe, "PuP: An Internetwork Architecture", XEROX Palo Alto Research Center, CSL-79-10, July 1979; also in IEEE Transactions on Communication, Volume COM-28, Number 4, April 1980.

[7] Braden, R., "NETRJS Protocol", RFC 740, NIC 42423, 22 November 1977. Also in [17].

[8] Bressler, B., "Remote Job Entry Protocol", RFC 407, NIC 12112, 16 October 72. Also in [17].

[9] Bressler, R., "Inter-Entity Communication -- An Experiment", RFC 441, NIC 13773, 19 January 1973.

[10] CCA, "Datacomputer Version 5/4 User Manual", Computer Corporation of America, August 1979.

[11] Clark, D., "Revision of DSP Specification", Local Network Note 9, Laboratory for Computer Science, MIT, 17 June 1977.

[12] Cohen, D., "Specifications for the Network Voice Protocol", RFC 741, ISI/RR 7539, USC/Information Sciences Institute, March 1976.

[13] Cohen, D. and J. Postel, "Multiplexing Protocol", IEN 90, USC/Information Sciences Institute, May 1979. 
COMPASS, "Semi-Annual Technical Report", CADD-7603-0411, Massachusetts Computer Associates, 4 March 1976. Also as, "National Software Works, Status Report No. 1," RADC-TR-76-276, Volume 1, September 1976. And COMPASS. "Second Semi-Annual Report," CADD-7608-1611, Massachusetts Computer Associates, 16 August 1976.

[15] Crispin, M., "SUPDUP Protocol", RFC 734, NIC 41953, 7 October 1977. Also in [17].

[16] Feinler, E., "Internet Protocol Transition Workbook", Network Information Center, SRI International, March 1982.

[17] Feinler, E. and J. Postel, eds., "ARPANET Protocol Handbook", NIC 7104, for the Defense Communications Agency by SRI International, Menlo Park, California, Revised January 1978.

[18] Forgie, J., "ST - A Proposed Internet Stream Protocol", IEN 119, M.I.T. Lincoln Laboratory, September 1979.

[19] Forsdick, H., "CFTP", Network Message, Bolt Berenak and Newman, January 1982 .

[20] Harrenstien, K., "Name/Finger", RFC 742, NIC 42758, 30 December 1977. Also in [17].

[21] Harrenstien, K., V. White, and E. Feinler, "Hostnames Server", RFC 811, SRI International, March 1982.

[22] Harrenstien, K., and V. White, "Nicname/Whois", RFC 812, SRI International, March 1982.

[23] Haverty, J., "XNET Formats for Internet Protocol Version 4", IEN 158, October 1980 .

[24] Hinden, R., A. Sheltzer, "The DARPA Internet Gateway", RFC 823, September 1982 .

[25] Honeywell CISL, Internal Document, "AFSDSC Hyperchannel RPQ Project Plan".

[26] Honeywell CISL, Internal Document, "Multics MR11 PFS".

[27] Korb, John T., "A Standard for the Transmission of IP Datagrams Over Public Data Networks", RFC 877, Purdue University, September 1983. 
[28] Littauer, B., "A Host Monitoring Protocol", IEN 197, Bolt Berenak and Newman, September 1981.

[29] Macgregor, W., and D. Tappan, "The CRONUS Virtual Local Network", RFC 824, Bolt Beranek and Newman, 22 August 1982.

[30] Malis, A., "Logical Addressing Implementation Specification", BBN Report 5256, pp 31-36, May 1983.

[31] Metcalfe, R.M. and D.R. Boggs, "Ethernet: Distributed Packet Switching for Local Computer Networks", Communications of the ACM, 19 (7), pp 395-402, July 1976.

[32] NSW Protocol Committee, "MSG: The Interprocess Communication Facility for the National Software Works", CADD-7612-2411, Massachusetts Computer Associates, BBN 3237, Bolt Beranek and Newman, Revised 24 December 1976.

[33] Plummer, D., "An Ethernet Address Resolution Protocol or Converting Network Protocol Addresses to 48-bit Ethernet Addresses for Transmission on Ethernet Hardware", RFC 826, MIT LCS, November 1982 .

[34] Postel, J., "Active Users", RFC 866, USC/Information Sciences Institute, May 1983.

[35] Postel, J., "Character Generator Protocol", RFC 864, USC/Information Sciences Institute, May 1983.

[36] Postel, J., "Daytime Protocol", RFC 867, USC/Information Sciences Institute, May 1983.

[37] Postel, J., "Discard Protocol", RFC 863, USC/Information Sciences Institute, May 1983.

[38] Postel, J., "Echo Protocol", RFC 862, USC/Information Sciences Institute, May 1983.

[39] Postel, J., "File Transfer Protocol", RFC 765, IEN 149, USC/Information Sciences Institute, June 1980.

[40] Postel, J., "Internet Control Message Protocol - DARPA Internet Program Protocol Specification", RFC 792, USC/Information Sciences Institute, september 1981.

[41] Postel, J., "Internet Message Protocol", RFC 759, IEN 113, USC/Information Sciences Institute, August 1980. 
[42] Postel, J., "Name Server", IEN 116, USC/Information Sciences Institute, August 1979.

[43] Postel, J., "Quote of the Day Protocol", RFC 865, USC/Information Sciences Institute, May 1983.

[44] Postel, J., "Remote Telnet Service", RFC 818, USC/Information Sciences Institute, November 1982.

[45] Postel, J., "Simple Mail Transfer Protocol", RFC 821, USC/Information Sciences Institute, August 1982.

[46] Postel, J., "User Datagram Protocol", RFC 768 USC/Information Sciences Institute, August 1980.

[47] Postel, J., ed., "Internet Protocol - DARPA Internet Program Protocol Specification", RFC 791, USC/Information Sciences Institute, September 1981 .

[48] Postel, J., ed., "Transmission Control Protocol - DARPA Internet Program Protocol Specification", RFC 793, USC/Information Sciences Institute, september 1981.

[49] Postel, J., and K. Harrenstien, "Time Protocol", RFC 868, USC/Information Sciences Institute, May 1983.

[50] Postel, J., and J. Reynolds, "Telnet Protocol specification", RFC 854, USC/Information Sciences Institute, May 1983.

[51] Reed, D., "Protocols for the LCS Network", Local Network Note 3, Laboratory for Computer Science, MIT, 29 November 1976.

[52] Reynolds, J. and J. Postel, "Official Protocols", RFC 880, USC/Information Sciences Institute, October 1983.

[53] Rosen, E., "Exterior Gateway Protocol" RFC 827, Bolt Berenak and Newman, October 1982 .

[54] Skelton, A., S. Holmgren, and D. Wood, "The MITRE Cablenet Project", IEN 96, April 1979.

[55] Sollins, K., "The TFTP Protocol (Revision 2)", RFC 783, MIT/LCS, June 1981 .

[56] Solomon, M., L. Landweber, and D, Neuhengen, "The CSNET Name Server", Computer Networks, v.6, n.3, pp. 161-172, July 1982 . 
Documents

[57] Sproull, R., and E. Thomas, "A Networks Graphics Protocol", NIC 24308, 16 August 1974. Also in [17].

[58] "The Ethernet - A Local Area Network", Version 1.0, Digital Equipment Corporation, Intel Corporation, Xerox Corporation, September 1980 .

[59] The High Level Protocol Group, "A Network Independent File Transfer Protocol", INWG Protocol Note 86, December 1977.

[60] Whelan, D., "The Caltech Computer Science Department Network", 5052:DF:82, Caltech Computer Science Department, 1982.

[61] XEROX, "The Ethernet, A Local Area Network: Data Link Layer and Physical Layer Specification", X3T51/80-50, Xerox Corporation, Stamford, CT., October 1980.

[62] XEROX, "Internet Transport Protocols", XSIS 028112, Xerox Corporation, Stamford, Connecticut, December 1981. 


\begin{tabular}{|c|c|c|c|}
\hline & & ------ & \\
\hline$[\mathrm{AGM}]$ & Andy Malis & $\mathrm{BBN}$ & Malis@BBN-UNIX \\
\hline$[\mathrm{APS}]$ & Anita Skelton & MITRE & skelton@MITRE \\
\hline$[\mathrm{AP}]$ & Alan Parker & NRL & parker@NRL-CSS \\
\hline$[\mathrm{AV}]$ & Al Vezza & MIT & AV@MIT-XX \\
\hline$[\mathrm{AXG}]$ & Atul Garg & $\mathrm{HP}$ & --- none--- \\
\hline [BG5 ] & Bob Gilligan & SRI & Gilligan@SRI-KL \\
\hline [BML ] & Barry Leiner & ARPA & Leiner@USC-ISIA \\
\hline$[\mathrm{BXA}]$ & Bobby W. Allen & YPG & WYMER@OFF ICE \\
\hline$[\mathrm{CAK}]$ & Chris Kent & PURDUE & cak@PURDUE \\
\hline [CC2 ] & Chase Cotton & UDEL & Cotton@Udel-EE \\
\hline [ CJW2 ] & Cliff Weinstein & LL & cjw@LL-11 \\
\hline [CLH3 $]$ & Charles Hedrick & RUTGERS & Hedrick@RUTGERS \\
\hline$[\mathrm{CMR}]$ & Craig Rogers & ISI & Rogers@USC-ISIB \\
\hline [DAM1 ] & David A. Mosher & $\mathrm{UCB}$ & Mosher@BERKELEY \\
\hline$[\mathrm{DCP} 1]$ & David Plummer & $\mathrm{MIT}$ & $\mathrm{DCP} @ \mathrm{MIT}-\mathrm{MC}$ \\
\hline$[\mathrm{DCT}]$ & Dan Tappan & $\mathrm{BBN}$ & Tappan@BBNG \\
\hline [DDC2 ] & Dave Clark & MIT-LCS & Clark@MIT-Multics \\
\hline$[\mathrm{DHH}]$ & Doug Hunt & BBN & DHunt@BBN-Unix \\
\hline [DK2 ] & Dean B. Krafft & CORNELL & Dean@CORNELL \\
\hline [DLM1 ] & David Mills & LINKABIT & Mills@USC-ISID \\
\hline [DM11] & Dale McNeill & $\mathrm{BBN}$ & mcneill@BBN-Unix \\
\hline$[\mathrm{DPR}]$ & David Reed & MIT-LCS & DPR@MIT-XX \\
\hline [DSW] & Dan Whelan & Caltech & Dan@CIT-20 \\
\hline [EAK1] & Earl Killian & LLL & EAK@MIT-MC \\
\hline [EAT3 ] & Ed Taft & XEROX & Taft.PA@PARC-MAXC \\
\hline$[\mathrm{EBM}]$ & Eliot Moss & MIT & EBM@MIT-XX \\
\hline$[\mathrm{EC} 5]$ & Ed Cain & DCEC & cain@EDN-Unix \\
\hline [EF 5] & Ed Franceschini & NYU & Franceschini@NYU \\
\hline [EHP ] & Ed Perry & SRI & PerryeSRI-KL \\
\hline [FAS ] & Fred Segovich & Compion & fred@COMP ION-VMS \\
\hline [GEOF ] & Geoff Goodfellow & SRI & Geoff@DARCOM-KA \\
\hline [HCF 2] & Harry Forsdick & $\mathrm{BBN}$ & Forsdick@BBNG \\
\hline [ $\mathrm{HDCl}]$ & Horst Clausen & DFVLR & Clausen@USC-ISID \\
\hline [ HDW2 ] & Howard Wactlar & $\mathrm{CMU}$ & Wactlar@CMU-10B \\
\hline [ HH 6 ] & Heidi Heiden & DCA & Heiden@BBNC \\
\hline [ HM] & Hank Magnuski & --- & JOSE@PARC-MAXC \\
\hline [ JAKE ] & Jake Feinler & SRI & Feinler@SRI-KL \\
\hline [ JAR4 ] & Jim Rees & WASHINGTON & N JIMQWASHINGTON \\
\hline [ JAW3 ] & Jil Westcott & $\mathrm{BBN}$ & Westcott@BBNF \\
\hline [ JBP ] & Jon Postel & ISI & Postel@USC-ISIF \\
\hline$[\mathrm{JC} 11]$ & Jim Clifford & LANL & jrc@LANL \\
\hline$[\mathrm{JCM}]$ & Jeff Mogul & STANFORD & MoguleSU-SCORE \\
\hline [ JD21] & Jonathan Dreyer & $\mathrm{BBN}$ & JDreyeraBBN-Unix \\
\hline [ JDG ] & Jim Guyton & RAND & guyton@RAND-Unix \\
\hline [ JEM $]$ & Jim Mathis & SRI & Mathis@SRI-KL \\
\hline [ JFH2 ] & Jack Haverty & BBN & Haverty@BBN-Unix \\
\hline
\end{tabular}




\begin{tabular}{|c|c|c|c|}
\hline$[\mathrm{JGH}]$ & Jim Herman & $\mathrm{BBN}$ & Herman@BBN-Unix \\
\hline [ JO5 ] & John O'Donnell & YALE & ODonnell@YALE \\
\hline [ JRM1 ] & John Mullen & MITRE & Mullen@MITRE \\
\hline [ JSG5] & Jon Goodridge & $\mathrm{BBN}$ & jsg@BBN-UNIX \\
\hline$[\mathrm{JWF}]$ & Jim Forgie & LL & Forgie@BBNC \\
\hline$[\mathrm{JXS}]$ & Jeffrey R. Schwab & PURDUE & jrs@PURDUE \\
\hline$[\mathrm{JZS}]$ & Joanne Sattely & $\mathrm{CCA}$ & JZS@CCA \\
\hline$[\mathrm{KLH}]$ & Ken Harrenstien & SRI & KLH@NIC \\
\hline [KRS ] & Karen Sollins & $\mathrm{MIT}$ & Sollins@MIT-XX \\
\hline$[\mathrm{LB} 1]$ & Liudvikas Bukys & ROCHESTER & Bukys@ROCHESTER \\
\hline$[\mathrm{LCN}]$ & Lou Nelson & AEROSPACE & Lou@AEROSPACE \\
\hline$[\mathrm{LCS}]$ & Lou Schreier & SRI & Schreier@USC-ISID \\
\hline [ LH2 $]$ & Lincoln $\mathrm{Hu}$ & COLUMBIA & Hu@Columbia-20 \\
\hline [LLG ] & Larry Garlick & XEROX & Garlick@PARC-MAXC \\
\hline [ LM8 ] & Liza Martin & MIT-LCS & Martin@MIT-XX \\
\hline$[\mathrm{MBG}]$ & Michael Greenwald & MIT-LCS & Greenwald@MIT-Multics \\
\hline$[\mathrm{MB}]$ & Michael Brescia & $\mathrm{BBN}$ & Brescia@BBN-Unix \\
\hline [MCSJ $]$ & Mike StJohns & AFDSC & StJohns@MIT-MULTICS \\
\hline [MH12] & Mark Horton & $\mathrm{ATT}$ & mark@BERKELEY \\
\hline [MHS1] & Marvin Solomon & WISC & Solomon@UWISC \\
\hline [MJM2 ] & Mike Muuss & BRL & Mike@BRL \\
\hline [MO2 ] & Michael O'Brien & RAND & OBrien@RAND-Unix \\
\hline$[\mathrm{MRC}]$ & Mark Crispin & Stanford & Admin.MRC@SU-SCORE \\
\hline$[\mathrm{MXB}]$ & Mark Brown & USC & Mark@USC-ECLB \\
\hline$[\mathrm{MXR}]$ & Marshall Rose & Irvine & MRose.UCI@RAND-Relay \\
\hline [NC3 ] & J. Noel Chiappa & $\mathrm{MIT}$ & JNC@MIT-XX \\
\hline$[\mathrm{NM}]$ & Neil MacKenzie & RSRE & T45@USC-ISID \\
\hline$[\mathrm{NXK}]$ & Neil Katin & $\mathrm{HP}$ & hpda.neil@BERKELEY \\
\hline$[\mathrm{PK}]$ & Peter Kirstein & UCL & Kirstein@USC-ISIA \\
\hline [PM1 ] & Paul Mockapetris & ISI & Mockapetris@USC-ISIF \\
\hline [PS3 $]$ & Paal Spilling & NDRE & Paal@DARCOM-KA \\
\hline$[\mathrm{PXD}]$ & Pieter Ditmars & $\mathrm{BBN}$ & pditmars@BBN-UNIX \\
\hline$[\mathrm{PXN}]$ & Peter Nellessen & SIEMENS & crtvax!pn@CMU-CS-SPICE \\
\hline [RA11] & Rick Adams & $\mathrm{CCI}$ & rlgvax!ra@SEISMO \\
\hline$[\mathrm{RB} 6]$ & Richard Bisbey & ISI & Bisbey@USC-ISIB \\
\hline [RDB2 ] & Robert Bressler & $\mathrm{BBN}$ & Bressler@BBN-Unix \\
\hline [REK2 ] & Robert Kahn & ARPA & Kahn@USC-ISIA \\
\hline$[\mathrm{RF} 1]$ & Randy Frank & UTAH & Frank@UTAH-20 \\
\hline [RH 6] & Robert Hinden & $\mathrm{BBN}$ & Hinden@BBN-Unix \\
\hline$[\mathrm{RHT}]$ & Robert Thomas & $\mathrm{BBN}$ & BThomas@BBNG \\
\hline [RK1 ] & Richard Kovalcik & Honeywell & Kovalcik@MIT-MULTICS \\
\hline [RLB1 ] & Bob Brown & USRA & rlbeames-vmsb \\
\hline [RLH2 ] & Ronald L. Hartung & NSWC & ronenswc-wo \\
\hline [RR2 ] & Raleigh Romine & Teledyne & romine@SEISMO \\
\hline [RS23] & Russel Sandberg & WISC & root@UWISC \\
\hline [RTB ] & Bob Braden & UCLA & Braden@USC-ISIA \\
\hline$[\mathrm{SC} 3]$ & Steve Casner & ISI & Casner@USC-ISIB \\
\hline$[\mathrm{SGC}]$ & Steve Chipman & $\mathrm{BBN}$ & Chipman@BBNA \\
\hline
\end{tabular}


Assigned Numbers

RFC 870

People

$\begin{array}{llll}{[\mathrm{SK}]} & \text { Steve Kille } & \text { UCL } & \text { UKSAT@USC-ISID } \\ {[\mathrm{SXM}]} & \text { Scott Marcus } & \text { Spartacus } & --- \text { none--- } \\ {[\mathrm{TC} 4]} & \text { Tony Cincotta } & \text { DTNSRDC } & \text { tony@NALCON } \\ {[\text { WIM }]} & \text { William Macgregor } & \text { BBN } & \text { macg@BBN } \\ {[\mathrm{ZSU}]} & \text { Zaw-Sing Su } & \text { SRI } & \text { ZSu@SRI-TSC }\end{array}$




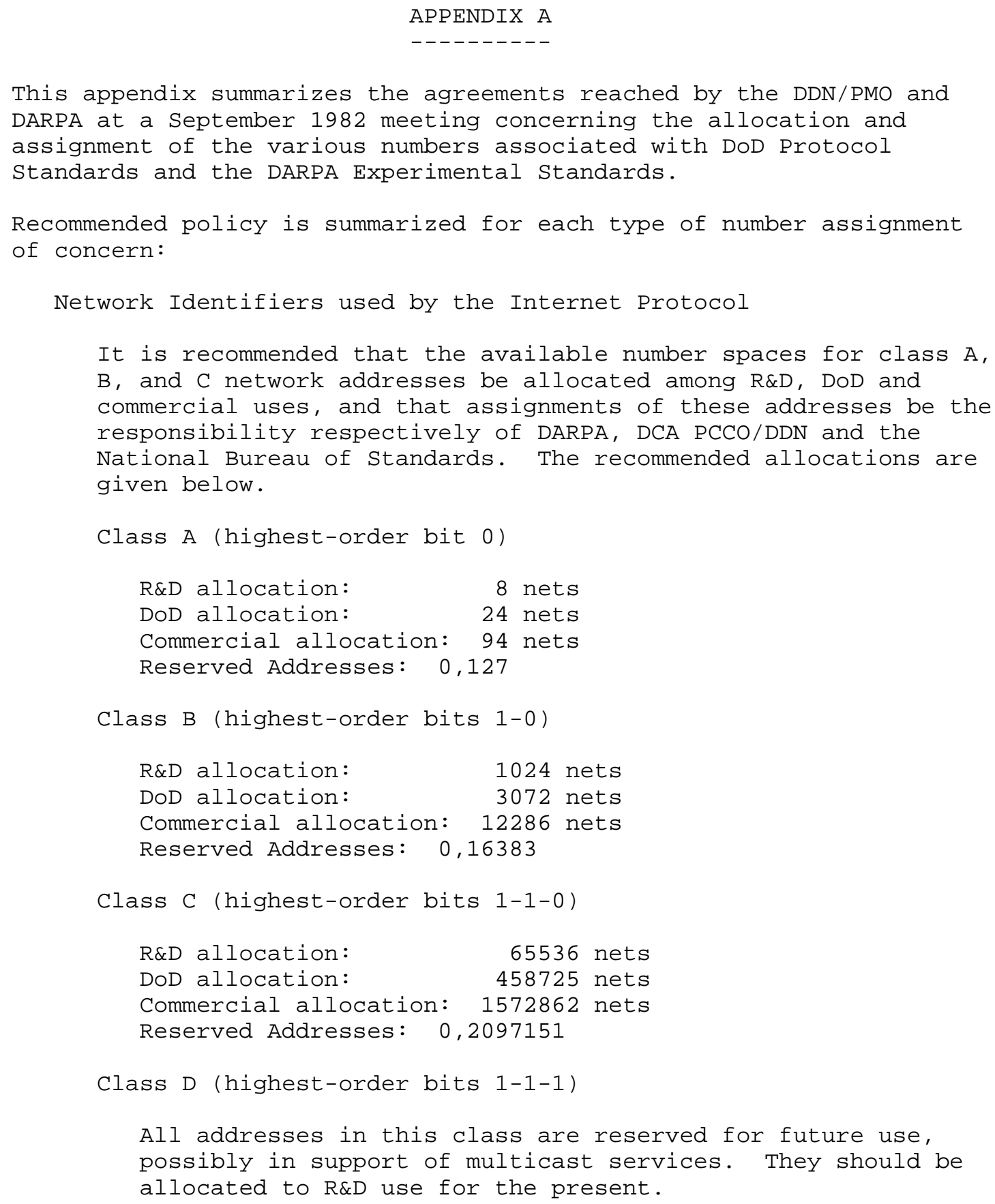


Within the R\&D community, it will be the policy that network identifiers will only be granted to applicants who show evidence that they are acquiring standard Bolt Beranek and Newman gateway software or have implemented or are acquiring a gateway meeting the Exterior Gateway Protocol requirements. Acquisition of the Berkeley BSD 4.2 UNIX software might be considered evidence of the latter.

Experimental networks which later become operational need not be renumbered. Rather, the identifiers could be moved from R\&D to DoD or Commercial status. Thus, network identifiers may change state among R\&D, DoD and commercial, but the number of identifiers allocated to each use should remain within the limits indicated above. To make possible this fluid assignment, it is recommended that the network identifier spaces not be allocated by simple partition but rather by specific assignment.

Protocol Identifiers

In general, all assignments will be made by the R\&D community, but any numbers which become R\&D, DoD, national or international standards will be marked as such in this RFC.

Protocol identifiers 0 and 255 are reserved.

95 protocol identifiers are allocated for assignment to DoD standards, 32 for R\&D use, and 127 for Commercial, national or international standards.

Port Numbers

A recommendation for allocation and assignment of port numbers is to be developed jointly by representatives of the ICCB and PSTP.

ARPANET Link Numbers

All unnecessary link number usage will be eliminated by joint effort of the ICCB, PSTP and BBN.

BBN will give consideration to the use of link numbers to promote interoperability among various ARPANET interfaces and report to the ICCB, PSTP and DDN/PMO. Examples of possible interoperability issues are:

(i) interoperability of 1822 and X.25 interfaces

(ii) interoperability of SIP and other interfaces

(iii) logical addressing or other special services 
IP Version Numbers

These numbers will be assigned only by the R\&D community for the purpose of exploring alternatives in internet protocol service expansion, such as inclusion of stream protocol (ST) services.

TCP, IP and Telnet Option Identifiers

These numbers will be assigned by the R\&D community. Any permanent or experimental assignments will be identified in the documents specifying those protcols.

Implementation:

This policy recommendation has not been fully implemented as yet. Currently, Joyce Reynolds is acting coordinator for all number assignments. 